\title{
Contact-Dependent Growth Inhibition Proteins in Acinetobacter baylyi ADP1
}

\author{
Eliana De Gregorio ${ }^{1} \cdot$ Eliana Pia Esposito ${ }^{2} \cdot$ Raffaele Zarrilli $^{2} \cdot$ Pier Paolo Di Nocera ${ }^{1}$
}

Received: 13 April 2018 / Accepted: 12 July 2018 / Published online: 17 July 2018

(c) The Author(s) 2018

\begin{abstract}
Bacterial contact-dependent growth inhibition (CDI) systems are two-partner secretion systems in which toxic CdiA proteins are exported on the outer membrane by cognate transporter $\mathrm{CdiB}$ proteins. Upon binding to specific receptors, the $\mathrm{C}$-terminal toxic (CT) domain, detached from CdiA, is delivered to neighbouring cells. Contacts inhibit the growth of not-self-bacteria, lacking immunity proteins co-expressed with CdiA, but promote cooperative behaviours in "self" bacteria, favouring the formation of biofilm structures. The Acinetobacter baylyi ADP1 strain features two CdiA, which differ significantly in size and have different CT domains. Homologous proteins sharing the same CT domains have been identified in A. baumannii. The growth inhibition property of the two A. baylyi CdiA proteins was supported by competition assays between wild-type cells and mutants lacking immunity genes. However, neither protein plays a role in biofilm formation or adherence to epithelial cells, as proved by assays carried out with knockout mutants. Inhibitory and stimulatory properties may be similarly uncoupled in A. baumannii proteins.
\end{abstract}

\section{Introduction}

Gram-negative bacteria exploit six secretion systems (types I-VI) to secrete proteins outside the cell [8]. Most secretion machineries are complex structures which span both the inner membrane (IM) and the outer membrane (OM). In the type $\mathrm{V}$ secretion system, proteins cross the IM through the Sec system and subsequently the OM either alone, in the auto-transporter pathway, or assisted by dedicated proteins, in the two-partner secretion (TPS) pathway [17]. The secreted proteins may remain onto the $\mathrm{OM}$, be released into the extracellular milieu, or be injected into target cells. Secreted proteins are involved in different processes which include adhesion to cells or abiotic surfaces, iron acquisition, invasion of eukaryotic cells, environmental adaptation.

Electronic supplementary material The online version of this article (https://doi.org/10.1007/s00284-018-1540-y) contains supplementary material, which is available to authorized users.

Eliana De Gregorio

edegrego@unina.it

1 Dipartimento di Medicina Molecolare e Biotecnologie Mediche, Università Federico II, Via Sergio Pansini 5, 80131 Naples, Italy

2 Dipartimento di Sanità Pubblica, Università Federico II, Via Sergio Pansini 5, 80131 Naples, Italy
Many are weapons used to prey upon non-self-bacteria. In type VI secretion systems, proteins evolutionarily related to phage tails components assemble contractile tubules which deliver killing proteins to adjacent cells [4].

In the peculiar TPS systems known as contact-dependent growth inhibition (CDI), CdiA proteins are exported on the outer membrane by cognate $\mathrm{CdiB}$ proteins and stop upon contact the growth of neighbouring bacteria $[1,16]$. The C-terminal domain of CdiA (CdiA-CT) proteins contains a toxin activity delivered to targeted cells to inhibit their growth. Immunity CDI proteins neutralize toxin activity in CDI + bacteria. CDI systems had been identified in several Gram-negative bacteria, and multiple CdiA/CdiI toxin/ antitoxin systems had been described in the same bacterial species $[16,20]$. CdiA proteins promote also cooperative interactions between isogenic CDI + cells, facilitating biofilm formation. CDI-dependent cell-cell adhesion had been observed in Escherichia coli [1], Xylella fastidiosa [14], Xanthomonas axonopodis [13] Burkholderia thailandensis [12], P. aeruginosa [20]. The same holds for the type IV secretion system (T6SS), which has been shown to play an active role in kin recognition and territorial behaviour by exporting self-recognition proteins in Proteus mirabilis [28].

Acinetobacter baylyi is an environmental non-pathogenic bacterium occasionally found responsible for opportunistic infections [6], amenable to genetic modifications because 
highly competent in natural transformation [11]. In recent years, A. baylyi had been extensively analysed because provided of an efficient T6SS [23, 27] able to kill neighbouring bacteria and promote the acquisition of DNA from targeted cells [7].

The analysis of the complete genome sequence [3] revealed that the A. baylyi ADP1 strain potentially encodes two large surface proteins of 2000 and 3711 amino acids (orfs 2784 and 940, respectively), which were annotated as filamentous hemagglutinins.

The objectives of the present report were to (i) analyse the structural organization of these proteins and their relatedness to homologous proteins present in A. baumannii; (ii) demonstrate that they are components of two distinct CDI systems; (iii) analyse their role in biofilm formation and adherence to human pneumocytes.

\section{Materials and Methods}

\section{Construction of $A$. baylyi Mutants}

Acinetobacter baylyi ADP1 mutant strains were constructed by insertion of the kanamycin-resistance cassette into target genes as described previously [2]. Briefly, the kanR gene was PCR amplified from plasmid pCR2.1-TOPO (Thermo Fisher Scientific) using the $\mathrm{KmFw}$ and $\mathrm{KmRv}$ primers, and the upstream and the downstream regions of each target gene were amplified from ADP1 DNA using specific primer pairs. A nested overlap PCR was carried out with an Expand High Fidelity Taq DNA polymerase (Roche), using NestFw and NestRv primers to generate DNA fragments including the kanR cassette flanked by 400-700 bp of chromosomal regions upstream and downstream of the gene segment to be deleted. The nested overlap PCR was performed as previously described [9]. Transformation assays were performed as previously described [21]. Knockout deletions were verified in PCR experiments using primers CF and CR. Oligonucleotide primers are listed in Table S1. For biofilm assays, bacteria were grown in $\mathrm{LB}$ at 30 or $37^{\circ} \mathrm{C}$, or in brain heart infusion broth at $30{ }^{\circ} \mathrm{C}$.

\section{Competition Assays}

Overnight cultures were diluted in modified LB (10 g/l tryptone, $5 \mathrm{~g} / \mathrm{l}$ yeast extract, $0.5 \mathrm{~g} / \mathrm{l} \mathrm{NaCl}$ ) and incubated at $30{ }^{\circ} \mathrm{C}$, till an $\mathrm{A}_{600}$ of $\sim 1.0$ was reached. Cultures were then diluted to an $\mathrm{A}_{600}$ of 0.4 . For competition assays, $40 \mu \mathrm{l}$ of predator A. baylyi ADP1 were mixed with $4 \mu \mathrm{l}$ of prey cells and $20 \mu$ of the mixture were spotted on LB-agar plates. Plates were incubated at $30{ }^{\circ} \mathrm{C}$ for $4 \mathrm{~h}$. Then, spots were excised from the plate, placed in $500 \mu \mathrm{l}$ of PBS, serially diluted, and plated on LB agar containing $12.5 \mu \mathrm{g} / \mathrm{ml}$ kanamycin. Of each dilution, $100 \mu \mathrm{l}$ was spread for CFU count; $10 \mu \mathrm{l}$ was spotted to visualize the outcome of the competition assay. Experiments were performed in triplicate.

\section{RNA Analyses}

RNA was isolated from A. baylyi ADP1 cells at log, late$\log$, and stationary phases. To monitor expression levels of cdiA genes, RT-PCR analyses were carried out as previously described [24] using the oligonucleotides listed in Table S1. Transcript levels were normalized to 16S rRNA levels. Changes in transcript levels were determined by the relative quantitative method $(\Delta \Delta \mathrm{CT})$. Experiments were carried out in triplicate.

\section{Biofilm Formation Assay}

Quantitative biofilm formation on polystyrene surfaces was investigated as previously reported [29]. To quantify biofilm formation in glass tubes, cultures were grown overnight in 12-mm-diameter glass under static conditions for $72 \mathrm{~h}$ at $30^{\circ} \mathrm{C}$. Upon medium removal, tubes were washed three times with PBS. Subsequently, $5 \mathrm{ml}$ of $0.1 \%$ crystal violet was added for $15 \mathrm{~min}$ at room temperature, followed by rinsing with PBS. Tubes were photographed after air drying. For confocal laser scanning microscopy (CLSM) analyses, $\sim 2 \times 10^{5} \mathrm{CFU} / \mathrm{ml}$ of wild-type and mutant cells were added to cell culture plates containing glass coverslips and incubated in static conditions at $30^{\circ} \mathrm{C}$ for $72 \mathrm{~h}$. Biofilm images were recorded as previously described [9]. All experiments were performed in triplicate.

\section{Cell Adhesion Assays}

Adherence of A. baylyi strains to A549 cells (human type 2 pneumocytes) was determined as described previously [9], with minor modifications. In brief, $\sim 10^{5}$ A549 cells were infected with $\sim 10^{7}$ bacterial CFU and incubated for $60 \mathrm{~min}$ at $37{ }^{\circ} \mathrm{C}$ in $5 \% \mathrm{CO}_{2}(\mathrm{v} / \mathrm{v})$ atmosphere. After removal of nonadherent bacterial cells by washing with PBS, infected cells were lysed by the addition of $1 \mathrm{ml}$ distilled water and serial 10-fold dilutions were plated on LB agar to determine the number of CFU of adherent bacteria. Dilutions from harvested samples were seeded on LB agar plates and bacterial colony counts were assessed after overnight incubation at $37^{\circ} \mathrm{C}$. Each experiment was performed in triplicate.

\section{Statistical Analysis}

Data were analysed using GraphPad Prism Version 5. Differences between mean values were tested for significance by performing one-way ANOVA analysis followed by Dunnett's 
comparison test. A $P$ value $<0.001$ was considered to be statistically significant.

\section{In Silico Analyses}

The CT regions of the CdiA2784 and CdiA940 proteins were used as queries for homology searches at GenBank carried out against both complete and draft genomes databases classified as Acinetobacter (taxid:469). Protein domains were searched at the NCBI CDD (Conserved Domain Database) site (http://www.ncbi.nlm.nih.gov/Structure/cdd/ cdd.shtml). Protein alignments were generated with the CLUSTAL OMEGA program (https://www.ebi.ac.uk/Tools $/ \mathrm{msa} /$ clustalo/). The sequence types of $\mathrm{CDI}^{+}$strains were determined by querying either the genomes, or the pool of contig sequences of the strain of interest in FASTA format, against the A. baumannii MLST database (https://pubml st.org/bigsdb?db=pubmlst_abaumannii_pasteur_seqde f\&set_id=2\&page=sequence).

\section{Results and Discussion}

\section{Acinetobacter baylyi CDI Systems}

The A. baylyi ADP1 strain features two cdi gene clusters. Each includes three adjacent genes, which encode the $\mathrm{CdiB}$ transporter, the secreted CdiA protein and an immunity CdiI protein, respectively (Fig. 1a). RT-PCR analyses revealed that both $c d i$ gene clusters are transcribed (Fig. S1). The immunity genes $c d i \mathrm{I} 941$ and $c d i \mathrm{I} 2783$ are located at a distance of 1 and $12 \mathrm{bp}$ from the upstream $c$ diA genes, respectively, and hence are co-transcribed with them. CdiA2784 and CdiA940 share an extended signal peptide region (ESPR) domain at the $\mathrm{NH} 2$ side, which is recognized by the Sec-translocation machinery and eventually cleaved during the export through the IM, and a TPS domain involved in CdiA-CdiB interactions, but differ in length and organization. CdiA2784 features a domain of unknown function (DUF637, PF04830), and a PT (pre-toxin)-VENN domain (PF04829), located, as in CdiA from many bacterial species [20], immediately upstream of the CT region. The CdiA2784 CT region spans a Tox-REase-7 domain (PF15649). CdiA940 features a large repeat region, constituted by arrays of short repeats reminiscent of those found in $(B)$ pertussis filamentous hemagglutinin [18]. No toxin domain was recognized at the $\mathrm{COOH}$ terminus of CdiA940 at the NCBI Conserved Domain Database.

Homology searches showed that the two proteins were perfectly conserved in the few unfinished $A$. baylyi genomes deposited at GenBank (data not shown). CdiA2784 exhibits similarity to CdiA from $A$. baumannii reference strains ACICU and ATCC19606 (Fig. 1b), which carry different
CT. Proteins exhibiting $62 \%$ identity to CdiA2784 and carrying similar CT regions (78\% identity) were identified in several A. baumannii strains belonging to different lineages, including the epidemic sequence type (ST) 25 [26]. Some of these strains are shown in Fig. 1b. CdiA940 exhibited $41 \%$ identity to a large CdiA encoded by the A. baumannii Ab120-H2 strain [21], but $71 \%$ identity to large CdiA encoded by the same A. baumannii strains hosting CdiA2784 homologs. Noteworthy, these proteins feature the same CT region of CdiA940 (Fig. 1b). Sequence alignments of CdiA$\mathrm{CT}$ regions are reported in figure $\mathrm{S} 2$. Data shown suggest that both A. baylyi cdiA genes may have been acquired from the same A. baumannii cell.

\section{Growth Competition Experiments}

CdiA proteins have been shown to have two opposite effects on neighbouring cells. They may inhibit the growth of nonself-bacteria, which lack immunity proteins antagonizing their toxins, but may also stimulate self-bacteria to build up biofilm structures. We asked whether the two different A. baylyi CdiA were able to perform both functions. A. baylyi cells should be self-protected against the toxic action of CdiA940 and CdiA2784 by the immunity proteins CdiI941 and CdiI2783 (Fig. 1). To validate the functioning of the two hypothesized toxin-antitoxin systems, we obtained deletion derivatives, in which the antitoxin $c d i \mathrm{I} 941$ and $c d i \mathrm{I} 2783$ genes were knocked out by the insertion of the kanamycin gene cassette [2]. The $\Delta 941$ and $\Delta 2783$ mutants were used as prey in competition experiments against tenfold excess of wild-type predator A. baylyi cells. As shown in Fig. 2, the growth of the antitoxin-minus $\Delta 941$ and $\Delta 2783$ cells was significantly inhibited by wild-type toxin-producers cells. For each experiment, the number of prey cells survived to competition was reported. Growth competition between mutant prey cells and non-toxic, mock predator $E$. coli cells were carried out as control, to rule out that 10-fold excess of predator cells, by reducing nutrients availability, may lower prey's CFU in a non-specific way.

\section{Biofilm Formation and Adherence to A549 Human Bronchial Cells of cdiA Mutants}

Next, we asked whether CdiA2784 and CdiA940 enhance biofilm formation, similarly to CdiA in other bacterial species $[1,12-14,20]$. Two mutants of the $c d i A 940$ gene were tested, in which DNA encoding residues 1-802 of CdiA940 (940- $\left.\Delta \mathrm{NH}_{2}\right)$, or the entire $c$ diA gene $(\Delta 940)$, was deleted. Biofilm formation was unaffected by the complete knockout of the $c d i \mathrm{~A} 940$ gene but was surprisingly fourfold enhanced in $940-\Delta \mathrm{NH}_{2}$ cells, which resulted phenotypically different from wild-type cells (Fig. 3). Growth rates of wild-type and 940- $\Delta \mathrm{NH}_{2}$ cells were identical, ruling out that changes in 
$\mathbf{a}$
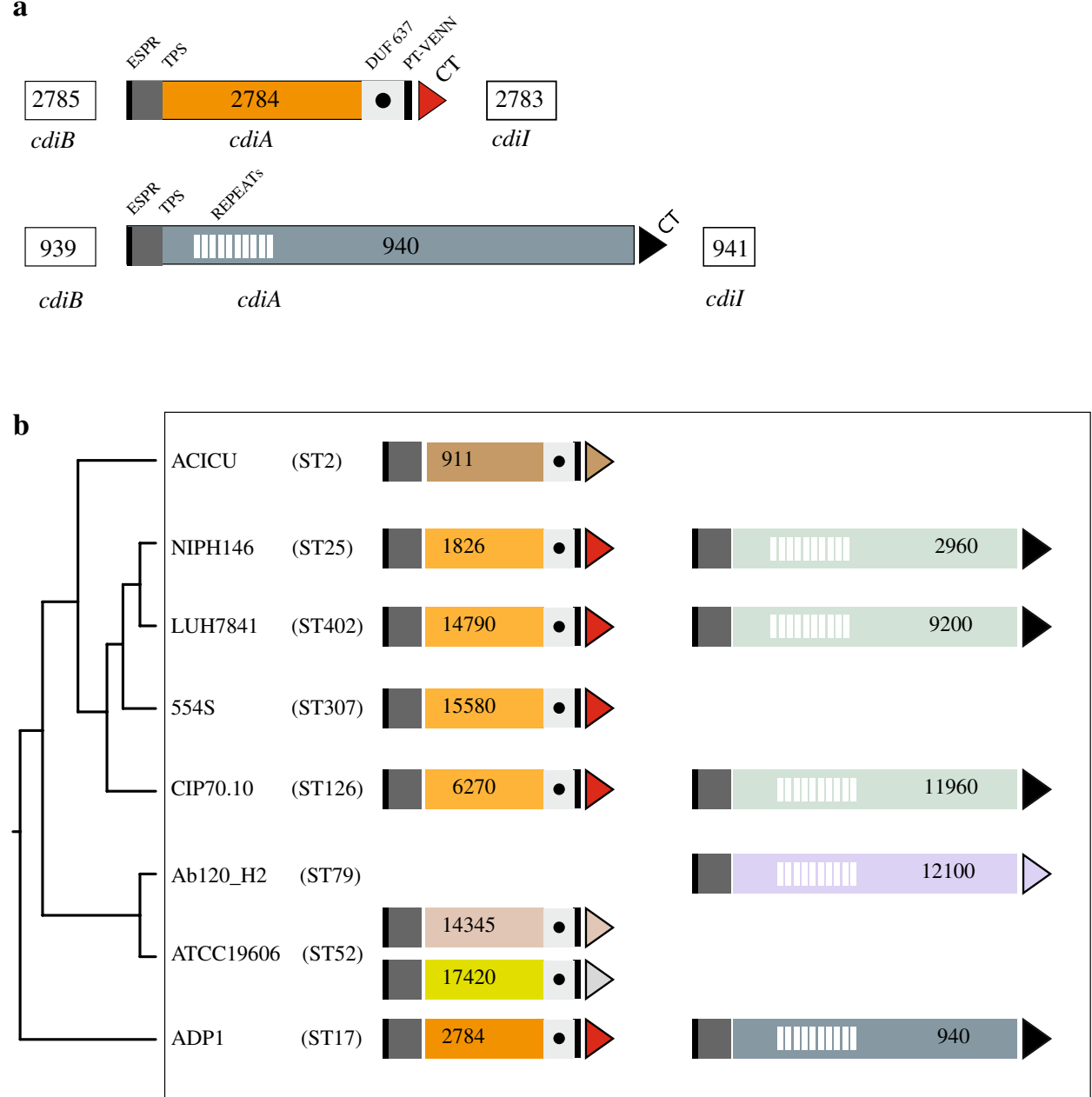

Fig. 1 Acinetobacter baylyi cdi genes. a The two CDI gene sets found in the ADP1 strain are sketched. Numbers refer to the orfs corresponding to $\mathrm{CdiB}$, CdiA, and CdiI. ESPR extended signal peptide region, TPS CdiB transporter binding domain, DUF637 domain of unknown function 637, PT VENN, pre-toxin VENN domain, $C T$ C-terminal toxic domain. b Representative A. baumannii strains featuring proteins homologous to A. baylyi CdiA are shown. For each, the ST (sequence type) is given in parenthesis. The cladogram results from the clustalW alignment of concatenated

morphology and ability to form biofilm were correlated to increased number of $940-\Delta \mathrm{NH}_{2}$ cells.

Similar mutants of the $c$ diA 2784 gene were obtained and assayed. The mutants $2784-\Delta \mathrm{NH}_{2}$ and $\Delta 2784$, in which DNA encoding residues $1-801$ of CdiA2784 or the entire $2784 c d i$ A gene was deleted, respectively, produced biofilm with similar efficiency as parental A. baylyi ADP1 cells (Fig. 3). Altogether, data rule out that CdiA proteins play a role in biofilm formation. In $940-\Delta \mathrm{NH}_{2}$, an aberrant CdiA protein could be fortuitously translated and reach the $\mathrm{OM}$, as shown for a $B$. pertussis hemagglutinin mutant lacking the TPS domain [10]. The increase in biofilm formation could have resulted from an altered surface presentation, due to allele sequences of the cpn60, fus, gltA, pyrG, recA, rplB, and rpoB gene segments from the indicated ST. GenBank strain references: ACICU (CP000865), NIPH146 (NZ_KB849308), LUH_7841 (orf 14790, JZBX01000012.1; orf 9200, JZBX01000006.1), 554S (NKXP01000136.1), CIP70.10 (NZ_LN865143.1), AbH120-A2 (NZ_CP009534.1), ATCC19606 (NZ_KB849990.1). Proteins from ATCC19606 and AbH120-A2 have been described [14, 21]. Genes and coding sequences were not drawn to scale. Homologous CT domains have the same colour. (Color figure online)

either membrane misplacement or misfolding of the truncated CdiA940. The mutant was not further analysed.

In light of the results obtained, we knocked out also $A$. baylyi ADP1 orf 2866, which corresponds to the biofilm associated protein (bap) gene. BAP is a surface protein proved crucial for biofilm formation in A. baumannii [19]. BAP has similarly a significant role in the process of biofilm formation in A. baylyi (Fig. 3).

We monitored whether the loss of CdiA or BAP proteins could interfere with the ability of A. baylyi ADP1 cells to interact with A549 human bronchial cells. No significant differences were observed in the ability of CdiA and BAP mutants to adhere to A549 cells in comparison with 
Fig. 2 Growth competition assays. $\Delta 941$ (a) and $\Delta 2783$ (b) cells were incubated $4 \mathrm{~h}$ at $30^{\circ} \mathrm{C}$ alone or mixed with a 10 -fold excess of either $A$. baylyi ADP1 or E. coli JM109 cells before plating (see "Materials and Methods" for details). Images correspond to one representative experiment from three independent assays done with different cultures of prey and predator cells a

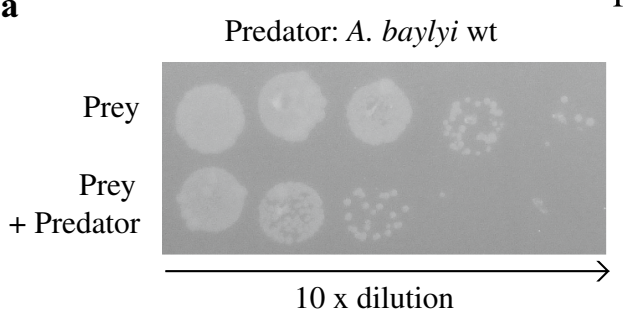

Prey: 941

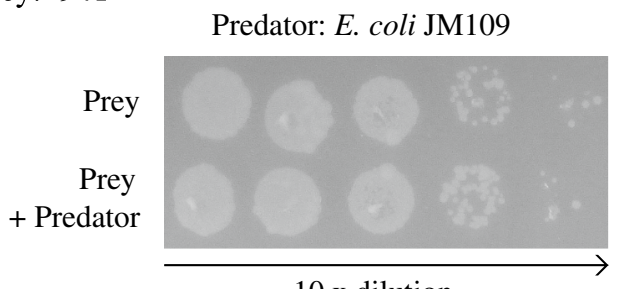

$10 \mathrm{x}$ dilution

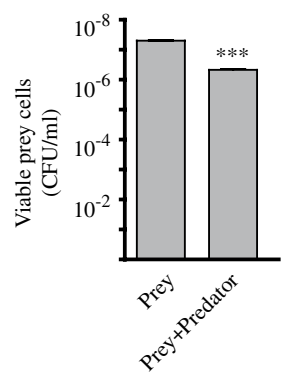

b

Predator: A. baylyi wt
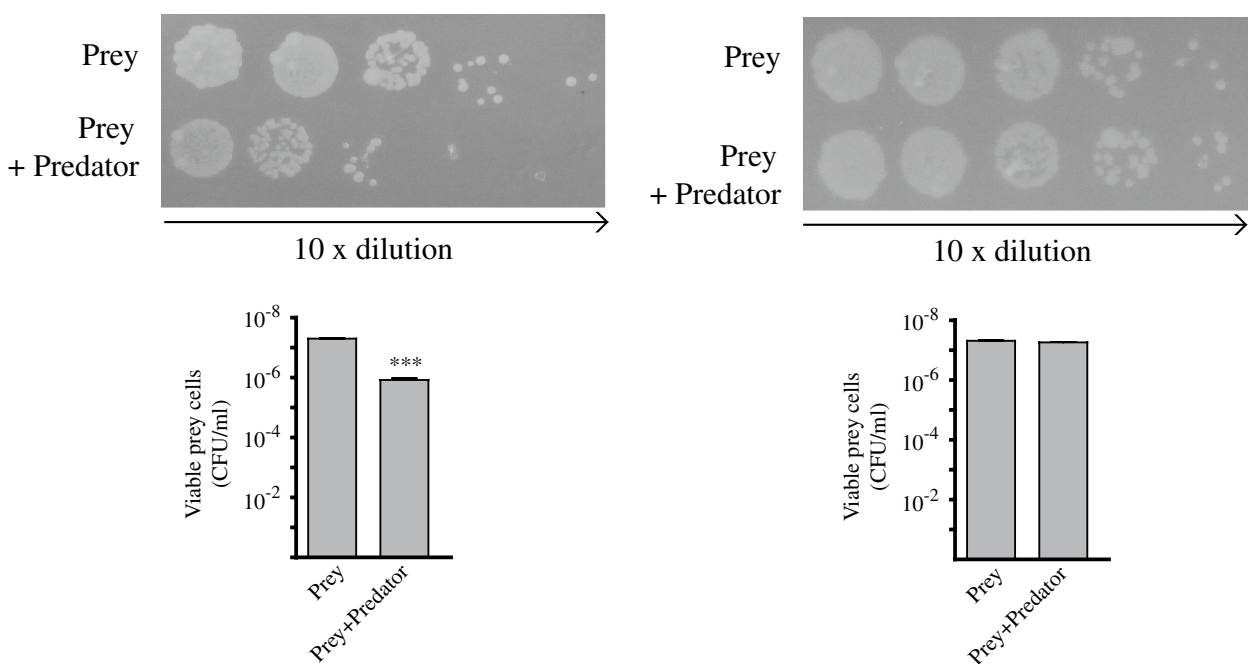

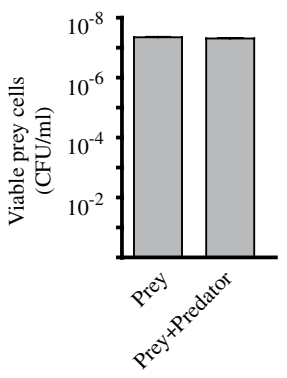

Prey: 2783

Predator: E. coli JM109

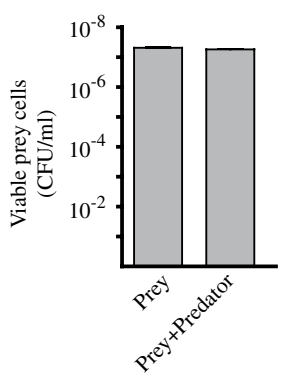

wild-type A. baylyi ADP1 cells (Fig. 4). The length of the protruding structures formed by the A. baylyi (1725 aa) and the A. baumannii ( 8200 aa) BAP on the OM could plausibly explain why only the A. baumannii BAP stimulated bacterial adhesion to human epithelial cells [5].

\section{Conclusions}

CdiA2784 and CdiA940 seem to function only as killing effectors since their absence does not impinge on the ability of $A$. baylyi cells to aggregate in biofilm structures. The finding that inhibitory and stimulatory properties are not associated in A. baylyi CdiA, as observed in most analogous proteins $[1,12-14,20]$, is of interest, and foreseeable for the properties of A. baumannii CDI systems. Data are in agreement with a previous study [22], showing that the AbfhaB CdiA expressed by the A. baumannii AbH120-A2 strain (Fig. 1b) was crucial for adhesiveness to A549 cells but had no role in biofilm formation. The adhesiveness to A549 cells may reflect intrinsic differences between AbfhaB and CdiA940, or the presence of auxiliary factors enhancing the AbfhaB-dependent adhesion to eukaryotic cells of AbH120-A2.

The reason why BAP, but not CdiA proteins, stimulates biofilm formation is not known and merits further investigation. In A. baumannii, biofilm formation process is associated to the selective expression and regulation of a myriad of genes [25]. Taking into account that $c d i$ are accessory genes, while bap genes are conserved 


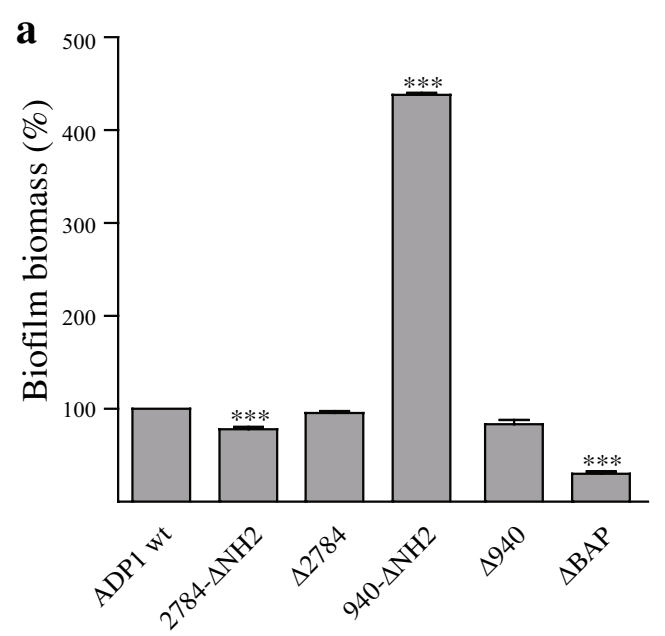

b
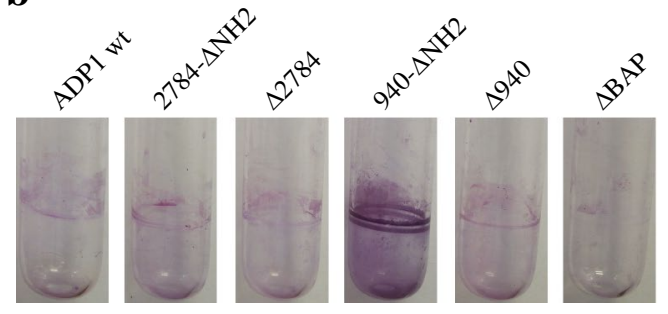

Fig. 3 Biofilm formation of A. baylyi ADP1 cells, CdiA, and BAP mutants. a Quantitation of biofilm formation in 96 multi-well plates. Data are the means of three independent assays and presented as mean \pm SEM,$P<0.001$. Asterisks indicate statistically significant $(P<0.001)$ differences versus $A$. baylyi ADP1 strain. b Representa-

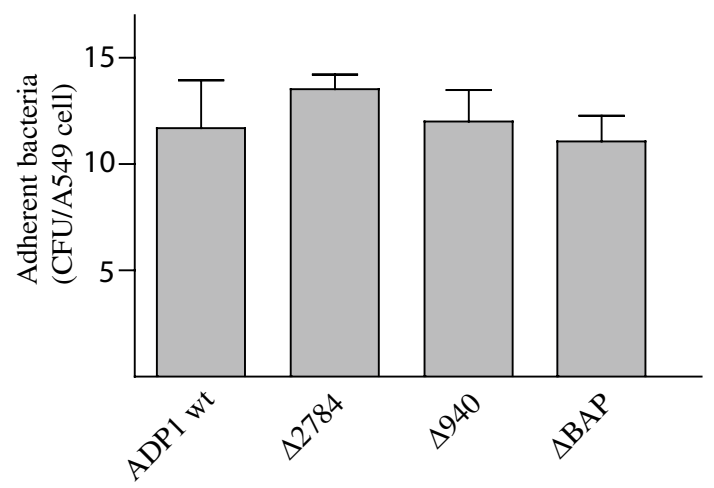

Fig. 4 Adherence of A. baylyi ADP1 cells, CdiA and BAP mutants to A549 bronchial epithelial cells. The amount of cell surface-associated bacteria after 60 -min incubation at $37{ }^{\circ} \mathrm{C}$ is shown. Asterisks denote statistically significant $(P<0.001)$ differences in the degree of cell adhesion

component of Acinetobacter genomes [9], it is tempting to speculate that BAP proteins may be co-regulated with other proteins involved in biofilm formation in Acinetobacter.

c
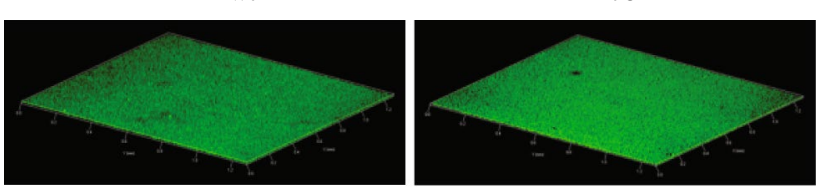

$\Delta 940$

$\triangle \mathrm{BAP}$
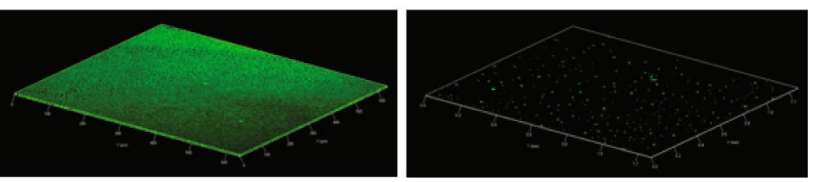

d

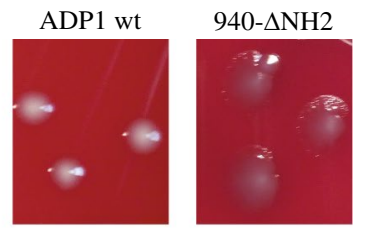

tive images of crystal violet (CV)-stained biofilms formed inside glass tubes. c Representative photographs of CLSM assays. Threedimensional images are shown $\mathbf{d}$ Colonies formed by $940-\Delta \mathrm{NH}_{2}$ and wt A. baylyi ADP1 cells

Acknowledgements We thank Prof. Paolo Visca for providing A. baylyi ADP1 strain. This research was partly supported by a Grant "Finanziamento della Ricerca di Ateneo" assigned by "Federico II" University of Naples, Italy, to PPDN and RZ.

\section{Compliance with Ethical Standards}

Conflict of interest The authors declare no conflict of interest.

Open Access This article is distributed under the terms of the Creative Commons Attribution 4.0 International License (http://creativeco mmons.org/licenses/by/4.0/), which permits unrestricted use, distribution, and reproduction in any medium, provided you give appropriate credit to the original author(s) and the source, provide a link to the Creative Commons license, and indicate if changes were made.

\section{References}

1. Aoki SK, Pamma R, Hernday AD et al (2005) Contactdependent inhibition of growth in Escherichia coli. Science 309:1245-1248 
2. Aranda J, Poza M, Pardo BG et al (2010) A rapid and simple method for constructing stable mutants of Acinetobacter baumannii. BMC Microbiol 10:279

3. Barbe V, Vallenet D, Fonknechten $\mathrm{N}$ et al (2004) Unique features revealed by the genome sequence of Acinetobacter sp. ADP1, a versatile and naturally transformation competent bacterium. Nucleic Acids Res 32:5766-5779

4. Basler M (2015) Type VI secretion system: secretion by a contractile nanomachine. Phil Trans R Soc B 370:20150021

5. Brossard KA, Campagnari AA (2012) The Acinetobacter baumannii biofilm-associated protein plays a role in adherence to human epithelial cells. Infect Immun 80:228-233

6. Chen TL, Siu LK, Lee YT et al (2008) Acinetobacter baylyi as a pathogen for opportunistic infection. J Clin Microbiol 46:2938-2944

7. Cooper RM, Tsimring L, Hasty J (2017) Inter-species population dynamics enhance microbial horizontal gene transfer and spread of antibiotic resistance. Elife 6:e25950

8. Costa TR, Felisberto-Rodrigues C, Meir A et al (2015) Secretion systems in Gram-negative bacteria: structural and mechanistic insights. Nat Rev Microbiol 13:343-359

9. De Gregorio E, Del Franco M, Martinucci M et al (2015) Biofilm-associated proteins: news from Acinetobacter. BMC Genom 16:933

10. Delattre AS, Saint N, Clantin B et al (2011) Substrate recognition by the POTRA domains of TpsB transporter FhaC. Mol Microbiol 81:99-112

11. Elliott KT, Neidle EL (2011) Acinetobacter baylyi ADP1: transforming the choice of model organism. IUBMB Life 63:1075-1080

12. Garcia EC, Anderson MS, Hagar JA et al (2013) Burkholderia $\mathrm{BcpA}$ mediates biofilm formation independently of interbacterial contact-dependent growth inhibition. Mol Microbiol 89:1213-1225

13. Gottig N, Garavaglia BS, Garofalo CG et al (2009) Filamentous hemagglutinin-like protein of Xanthomonas axonopodis pv. citri, the phytopathogen responsible for citrus canker, is involved in bacterial virulence. PLoS ONE 4:e4358

14. Guilhabert MR, Kirkpatrick BC (2005) Identification of Xylella fastidiosa antivirulence genes: hemagglutinin adhesins contribute a biofilm maturation to $X$. fastidiosa and colonization and attenuate virulence. Mol Plant Microbe Interact 18:856-868

15. Harding CM, Pulido MR, Di Venanzio G et al (2017) Pathogenic Acinetobacter species have a functional type I secretion system and contact-dependent inhibition systems. J Biol Chem 292:9075-9087
16. Hayes CS, Aoki SK, Low DA (2010) Bacterial contact-dependent delivery systems. Annu Rev Genet 44:71-90

17. Jacob-Dubuisson F, Guérin J, Baelen S et al (2013) Two-partner secretion: as simple as it sounds? Res Microbiol 164:583-595

18. Kajava AV, Cheng N, Cleaver R et al (2001) Beta-helix model for the filamentous haemagglutinin adhesin of Bordetella pertussis and related bacterial secretory proteins. Mol Microbiol 42:279-292

19. Loehfelm TW, Luke NR, Campagnari AA (2008) Identification and characterization of an Acinetobacter baumannii biofilm-associated protein. J Bacteriol 190:1036-1044

20. Mercy C, Ize B, Salcedo SP et al (2016) Functional characterization of Pseudomonas contact dependent growth inhibition (CDI) systems. PLoS ONE 11:e0147435

21. Metzgar D, Bacher JM, Pezo V et al (2004) Acinetobacter sp. ADP1: an ideal model organism for genetic analysis and genome engineering. Nucleic Acids Res 32:5780-5790

22. Pérez A, Merino M, Rumbo-Feal S et al (2016) The FhaB/FhaC two-partner secretion system is involved in adhesion of Acinetobacter baumannii AbH12O-A2 strain. Virulence 8:959-974

23. Repizo GD, Gagné S, Foucault-Grunenwald ML et al (2015) Differential role of the T6SS in Acinetobacter baumannii virulence. PLoS ONE 10:e0138265

24. Roscetto E, Angrisano T, Costa V et al (2012) Functional characterization of the RNA chaperone Hfq in the opportunistic human pathogen Stenotrophomonas maltophilia. J Bacteriol 194:5864-5874

25. Rumbo-Feal S, Gómez MJ, Gayoso C et al (2013) Whole Transcriptome analysis of Acinetobacter baumannii assessed by RNAsequencing reveals different mRNA expression profiles in biofilm compared to planktonic cells. PLoS ONE 8:e72968

26. Sahl JW, Del Franco M, Pournaras S et al (2015) Phylogenetic and genomic diversity in isolates from the globally distributed Acinetobacter baumannii ST25 lineage. Sci Rep 5:15188

27. Weber BS, Miyata ST, Iwashkiw JA et al (2013) Genomic and functional analysis of the type VI secretion system in Acinetobacter. PLoS ONE 8:e55142

28. Wenren LM, Sullivan NL, Cardarelli L et al (2013) Two independent pathways for self-recognition in Proteus mirabilis are linked by type VI-dependent export. MBio 4:e00374-e00313

29. Withers R, Doherty GP, Jordan M et al (2014) AtfA, a new factor in global regulation of transcription in Acinetobacter spp.. Mol Microbiol 93:1130-1143 